\title{
Reducibility for a Class of Almost-Periodic Differential Equations with Degenerate Equilibrium Point under Small Almost-Periodic Perturbations
}

\author{
Wenhua Qiu ${ }^{1,2}$ and Jianguo $S i^{1}$ \\ ${ }^{1}$ School of Mathematics, Shandong University, Jinan, Shandong 250100, China \\ ${ }^{2}$ School of Mathematics and Statistics, Zaozhuang University, Zaozhuang, Shandong 277160, China
}

Correspondence should be addressed to Jianguo Si; sijgmath@yahoo.com.cn

Received 18 July 2013; Revised 10 October 2013; Accepted 13 October 2013

Academic Editor: Svatoslav Staněk

Copyright (C) 2013 W. Qiu and J. Si. This is an open access article distributed under the Creative Commons Attribution License, which permits unrestricted use, distribution, and reproduction in any medium, provided the original work is properly cited.

\begin{abstract}
This paper focuses on almost-periodic time-dependent perturbations of an almost-periodic differential equation near the degenerate equilibrium point. Using the KAM method, the perturbed equation can be reduced to a suitable normal form with zero as equilibrium point by an affine almost-periodic transformation. Hence, for the equation we can obtain a small almost-periodic solution.
\end{abstract}

\section{Introduction and Main Result}

Reducibility of nonautonomous finite-dimensional systems with quasiperiodic coefficients has basic importance in the analysis of dynamical systems; see $[1,2]$. Unfortunately, we cannot guarantee in general such reducibility. In the last years, establishing the reducibility of finite-dimensional systems by means of the KAM tools is an active field of research, and many authors are devoted to the study of reducibility of such systems. In 1996, Jorba and Simó [3] considered reducibility of the following nonlinear quasiperiodic system near an elliptic equilibrium point:

$$
\begin{array}{r}
\dot{x}=(A+\epsilon Q(t, \epsilon)) x+\epsilon g(t, \epsilon)+h(x, t, \epsilon), \\
x \in \mathbb{R}^{d},
\end{array}
$$

where $A$ is assumed to be elliptic. $Q(t, \epsilon), g(t, \epsilon)$ and $h(x, t, \epsilon)$ depend on time in a quasiperiodic way with basic frequencies $\left(\omega_{1}, \ldots, \omega_{r}\right), h(x, \omega t)=O\left(x^{2}\right)$ as $x \rightarrow 0$. Under a nondegenerate condition and a nonresonant condition, using KAM iteration they proved that for most sufficiently small $\epsilon$ by an affine quasiperiodic transformation the system (1) is reducible to the following form:

$$
\dot{x}=A_{*}(\epsilon) x+h_{*}(x, t, \epsilon), \quad x \in \mathbb{R}^{d},
$$

where $A_{*}$ is a constant matrix close to $A$ and $h_{*}(x, t, \epsilon)=$ $O\left(x^{2}\right)(x \rightarrow 0)$ is a high-order term close to $h$. Therefore, the system (1) has a quasiperiodic solution near the zero equilibrium point. Some similar results were obtained in [4].

Recently, Xu and Jiang [5] considered the following nonlinear quasiperiodic differential equation:

$$
\dot{x}=x^{2 n+1}+h(x, \omega t)+f(x, \omega t), \quad x \in \mathbb{R},
$$

where $n \geq 0$ is an integer, $h=O\left(x^{2 n+2}\right)(x \rightarrow 0)$ is a higher-order term, $f$ is a small perturbation term, and $h$ and $f$ are all real analytic in $x$ and $t$, quasiperiodic in $t$ with frequency $\omega$. Under the Diophantine condition, when $f$ is sufficiently small the differential equation (3) can be reduced to a suitable normal form with zero as equilibrium point by an affine quasiperiodic transformation, so it has a quasiperiodic solution near zero.

In 1996, Xu and You [6] considered the following linear almost-periodic differential equation:

$$
\dot{x}=[A+\epsilon Q(t)] x, \quad x \in \mathbb{R}^{d},
$$

where $A$ is a constant $d \times d$ matrix with different eigenvalues $\lambda_{1}, \ldots, \lambda_{d}$ and $Q(t)$ is a $d \times d$ almost-periodic matrix with the frequency $\omega=\left(\omega_{1}, \omega_{2}, \ldots\right)$. Under some small divisor condition, they proved that, for most sufficiently small $\epsilon$ the 
system (4) is reducible to the form $\dot{y}=B y$ by an affine almostperiodic transformation.

By the above inspired works $[5,6]$, we consider the following nonlinear almost-periodic differential equation:

$$
\dot{x}=(A+\epsilon a(t)) x^{2 n+1}+h(x, t, \epsilon)+f(x, t, \epsilon), \quad x \in \mathbb{R},
$$

where $n \geq 0$ is an integer, $A$ is a positive number, $\epsilon$ is a small parameter, $h$ is a higher-order term, and $f$ is a small perturbation term. Under some suitable conditions, we show that the differential equation (5) can be reduced to a suitable normal form with zero as equilibrium point by an affine almost-periodic transformation, so it has an almost-periodic solution near zero.

For our purpose, we first introduce some definitions and notations.

Definition 1. The function $f(t)$ is called a quasiperiodic function of $t$ with frequencies $\omega_{1}, \omega_{2}, \ldots, \omega_{m}$, if there is a function $F(\theta)=F\left(\theta_{1}, \theta_{2}, \ldots, \theta_{m}\right)$, which is $2 \pi$-periodic in all its arguments $\theta_{i}(i=1, \ldots, m)$, such that $f(t)=F(\omega t)=$ $F\left(\omega_{1} t, \omega_{2} t, \ldots, \omega_{m} t\right)$.

If $F(\theta)$ is analytic on a strip domain $\mathbb{\mathbb { T }}_{s}=\left\{\theta \in \mathbb{C}^{m} / 2 \pi \mathbb{Z}^{m} \mid\right.$ $\left.\left|\operatorname{Im} \theta_{j}\right| \leq s, j=1,2, \ldots, m\right\}$, we say that $f(t)$ is analytic quasiperiodic on $\mathbb{T}_{s}$. Expand $f(t)$ as a Fourier series $f=$ $\sum_{k \in \mathbb{Z}^{m}} f_{k} e^{i\langle k, \theta\rangle}$, where $i=\sqrt{-1}$ and $\theta=\omega t$. Define $\|f\|_{\mathbb{T}_{s}}=$ $\sum_{k \in \mathbb{Z}^{m}}\left|f_{k}\right| e^{s|k|}$.

Write $D(0, r)=\{x \in \mathbb{C}|x| \leq r\}$ and $\Delta_{r, s}=D(0, r) \times \mathbb{T}_{s}$.

Definition 2. Let $P(x, t)$ be real analytic in $x$ and t on $\Delta_{r, s}$, and let $P(x, t)$ be quasiperiodic with respect to $t$ with the frequency $\omega$. Then $P$ can be expanded as a Fourier series as follows:

$$
P(x, t)=\sum_{k \in \mathbb{Z}^{m}} P_{k}(x) e^{i\langle k, \theta\rangle} .
$$

Define a norm by

$$
\|P\|_{\Delta_{r, s}}=\sum_{k \in \mathbb{Z}^{m}}\left|P_{k}\right|_{r} e^{|k| s}
$$

where $P_{k}(x)=\sum_{l=0}^{\infty} P_{l k} x^{l}$ and $\left|P_{k}\right|_{r}=\sup _{x \in D(0, r)} \sum_{l=0}^{\infty}\left|P_{l k}\right||x|^{l}$. It is easy to see that

$$
\left\|P_{1} P_{2}\right\|_{\Delta_{r, s}} \leq\left\|P_{1}\right\|_{\Delta_{r, s}} \cdot\left\|P_{2}\right\|_{\Delta_{r, s}} .
$$

Definition 3 (see [7]). A function $\Delta$ is called an approximation function if it satisfies the following:

$(1) \Delta:[0,+\infty) \rightarrow[1,+\infty), \Delta(0)=1$, and $\Delta$ is a nondecreasing function;

(2) $\log \Delta(t) / t$ is a decreasing function in $[0,+\infty)$;

(3) $\int_{0}^{\infty} \log \Delta(t) / t^{2}<+\infty$.

Obviously, any positive power of an approximation function is again an approximation function, so is the product of two such functions.
Definition 4 (see [8]). Suppose that $\mathbb{N}$ is the natural number set and $\tau$ is a set composed of the subset of $\mathbb{N}$. We say that $(\tau,[\cdot])$ is the finite spatial structure on $\mathbb{N}$ if $\tau$ satisfies the following:

(1) the empty set $\emptyset \in \tau$;

(2) if $\Lambda_{1}, \Lambda_{2} \in \tau$, then $\Lambda_{1} \cup \Lambda_{2} \in \tau$;

(3) $\bigcup_{\Lambda \in \tau} \Lambda=\mathbb{N}$, $[\emptyset]=0$ and $\left[\Lambda_{1} \cup \Lambda_{2}\right] \leq\left[\Lambda_{1}\right]+\left[\Lambda_{2}\right]$ ([.] is called a weight function defined in $\tau$ ).

Definition 5. Assume $k=\left(k_{1}, k_{2}, \ldots\right) \in \mathbb{Z}^{\infty}$, the set

$$
\begin{gathered}
\operatorname{supp} k=\left\{\left(i_{1}, i_{2}, \ldots, i_{n}\right) \mid k_{j} \neq 0 \text { as } j=i_{1}, i_{2}, \ldots, i_{n} ;\right. \\
\left.k_{j}=0 \text { as } j=\text { otherwise }\right\}
\end{gathered}
$$

is called the support set of $k$. Consider

$$
[k]=: \inf _{\operatorname{supp} k \subset \Lambda, \Lambda \in \tau}[\Lambda]
$$

is called the weight of $k$, and $|k|=: \sum_{i=1}^{\infty}\left|k_{i}\right|$.

Definition 6. If $Q(t)=\sum_{\Lambda \in \tau} Q_{\Lambda}(t)$ with $Q_{\Lambda}(t)$ is quasiperiodic function with the frequency $\omega_{\Lambda}=\left\{\omega_{i} \mid i \in \Lambda\right\}$, then $Q(t)$ is said to be almost-periodic function with the finite spatial structure $(\tau,[\cdot])$. If $\omega=\left(\omega_{1}, \omega_{2}, \ldots\right)$ is the biggest subset of $\cup \omega_{\Lambda}$ in the sense of integer modulus, then $\omega$ is called to be the frequency of $Q(t)$.

If almost-periodic function $Q(t)$ has a rapidly converging Fourier series expansion

$$
\mathrm{Q}(t)=\sum_{k \in Z^{\infty}} U_{k} e^{i\langle k, \omega t\rangle}
$$

where $\omega=\left(\omega_{1}, \omega_{2}, \ldots\right)$ is the frequency and $k=\left(k_{1}, k_{2}, \ldots\right)$ have only finitely many nonzero components, then $Q(t)$ is analytic in $t$.

Definition 7. Let $Q(t)=\sum_{\Lambda \in \tau} Q_{\Lambda}(t)$ with the frequency $\omega=$ $\left(\omega_{1}, \omega_{2}, \ldots\right)$. For $m \geq 0, s \geq 0$,

$$
\|\mathrm{Q}(t)\|\left\|_{m, s}=\sum_{\Lambda \in \tau} e^{m[\Lambda]}\right\| Q_{\Lambda}(t) \|_{\mathbb{T}_{s}}
$$

is called the weight norm of $Q(t)$ in the finite spatial structure $(\tau,[\cdot])$.

From Definition 7, we know that $|Q(t)| \leq\||| Q(t) \mid\|_{0, s} \leq$ $\|Q Q(t)\| \|_{m, s}$, for $m \geq 0, s \geq 0$.

Definition 8. Let $P(x, t)=\sum_{\Lambda \in \tau} P_{\Lambda}(x, t)$ with the frequency $\omega=\left(\omega_{1}, \omega_{2} \cdots\right)$, for $m \geq 0, s \geq 0$,

$$
\||| P(x, t) \mid\|_{m, \Delta_{r, s}}=\sum_{\Lambda \in \tau} e^{m[\Lambda]}\left\|P_{\Lambda}(x, t)\right\|_{\Delta_{r, s}}
$$

is called the weight norm of $P(x, t)$ in the finite spatial structure $(\tau,[\cdot])$. 
Let $Q(t)=\sum_{\Lambda \in \tau} Q_{\Lambda}(t)$ be almost-periodic function; then

$$
[Q]=\lim _{T \rightarrow \infty} \int_{-T}^{T} Q(t) d t
$$

is called the mean value of $Q(t)$. The existence of the limit can be found in [9].

Throughout this paper, we assume that the following hypotheses hold

(H1) the functions $a, h$, and $f$ are real analytic in all variables and almost-periodic in $t$ with common frequency vector $\omega=\left(\omega_{1}, \omega_{2}, \ldots\right)$; they also have the the finite spatial structure $(\tau,[\cdot])$;

(H2) $|\langle k, \omega\rangle| \geq \alpha / \Delta^{4}(|k|) \Delta^{4}([k]), k \in \mathbb{Z}^{\infty} \backslash\{0\}$, where $\alpha>0$ is a constant and $\Delta$ is an approximation function;

(H3) $h=\mathcal{O}\left(x^{2 n+2}\right)(x \rightarrow 0)$, where $n \geq 0$, and for fixed $m_{0}, s>0$, we have

$$
\||a(t)|\|_{m_{0}, s}<+\infty
$$

Now we are ready to state the main result of this paper.

Theorem 9. Suppose that conditions $(H 1)-(H 3)$ hold. Then there exists sufficiently small $\epsilon>0$, such that if

$$
\||| f \mid\|_{m_{0}, \Delta_{r, s}} \leq \epsilon,
$$

then there exists an affine real analytic almost-periodic transformation of the form $x=y+u(t)$ such that the differential equation (5) is changed to

$$
\dot{y}=A_{*} y^{2 n+1}+f_{*}(y, t),
$$

where $f_{*}(y, t)=O(y)$ as $y \rightarrow 0$. Moreover, $u$ is a real analytic almost-periodic in $t$ with

$$
\|u(t)\|_{0, s / 2}=O\left(\epsilon^{1 /(2 n+2)}\right),
$$

and $x=u(t)$ is also an almost-periodic solution of (5).

\section{Normal Form for an Almost-Periodic Equation with Parameters}

The proof of Theorem 9 is based on a norm form theorem for an almost-periodic equation with parameters. In this section, we first consider the following real almost-periodic differential equation with two parameters:

$$
\begin{aligned}
\dot{x}= & (A+\epsilon[a]) \xi^{2 n+1}-\lambda \\
& +(A+\epsilon a(t)) \Omega(\xi) x+P(x, t ; \xi, \lambda),
\end{aligned}
$$

where $[a]$ is the mean value of $a(t) . \lambda \in J=[-1,1], \xi \in I=$ $[-\delta, \delta]$ are parameters and $\Omega(\xi)=(2 n+1) \xi^{2 n}$.

Let $T \subset \mathbb{R}^{2}$ and $p=(\xi, \lambda)$. Denote by

$$
B(T, \sigma)=\left\{p \in \mathbb{C}^{2} \mid \operatorname{dist}(p, T) \leq \sigma\right\}
$$

the complex $\sigma$-neighborhood of $T$ in the two-dimensional complex space $\mathbb{C}^{2}$.

We will invoke the KAM iteration technique to prove the following normal form theorem.
Theorem 10. Let $M_{0}=B\left(I \times J, \sigma_{0}\right)$. Suppose that $\Omega$ and $P$ are analytic on $M_{0}$ and $\Delta_{r_{0}, s_{0}} \times M_{0}$, respectively. Let $\rho_{0}=s_{0} / 8$ and let $E_{0}>0$. Let $K_{0}>0$ such that $E_{0}=e^{-\rho_{0} K_{0}}$. There exists a sufficiently small $E_{0}>0$ such that if

$$
\begin{aligned}
& \frac{\epsilon_{0}}{E_{0} \sigma_{0}} \leq \frac{1}{4}, \quad \frac{\epsilon_{0}}{r_{0} \sigma_{0}} \leq \frac{1}{16}, \quad \frac{E_{0}}{r_{0}} \leq 1, \\
& \||| P\| \|_{m_{0}, \Delta_{r_{0}, s_{0}} \times M_{0}} \leq \epsilon_{0}=\frac{\alpha r_{0} E_{0}}{\Gamma\left(m_{0} / 2\right) \Gamma\left(\rho_{0}\right)},
\end{aligned}
$$

where

$$
\Gamma(\rho)=: \sup _{t \geq 0}\left[\Delta^{4}(t) e^{-\rho t}\right],
$$

then there exists a real $C^{\infty}$-smooth curve in $M_{0}$,

$$
T: \lambda=\lambda(\xi)=(A+\epsilon[a]) \xi^{2 n+1}+\widehat{N}(\xi), \quad \xi \in I .
$$

And for every $p \in T$, there exists an affine analytic almostperiodic transformation

$$
\Phi(\cdot, t ; p): x=y+u_{*}(t ; p),
$$

which changes (19) to

$$
\dot{y}=\Omega_{*}(p) y+P_{*}(y, t, p)
$$

with $P_{*}(y, t, p)=O\left(y^{2}\right)(y \rightarrow 0)$. Moreover,

$$
\begin{array}{r}
|\widehat{N}(\xi)| \leq 4 \epsilon_{0}, \quad\left|\Omega_{*}(p)-A \Omega(p)\right| \leq \frac{2 \epsilon_{0}}{r_{0}}, \\
\forall p=(\xi, \lambda) \in T .
\end{array}
$$

Furthermore, for $p \in T, x=u_{*}(t, p)$ is an analytic almostperiodic solution of the differential equation (19) with $\lambda=\lambda(\xi)$.

Lemma 11. Let $N(p)=(A+\epsilon[a]) \xi^{2 n+1}-\lambda+h(p), p=(\xi, \lambda) \epsilon$ $M$. Suppose that $h$ is real analytic on $M \subset \mathbb{C}^{2}$ with

$$
\left\|\mathscr{D}_{p} h\right\|_{M}=\left\|h_{\lambda}\right\|_{M}+\left\|h_{\xi}\right\|_{M}<\frac{1}{2} .
$$

Suppose that, on the domain $M, N(\xi, \lambda)=0$ determines implicitly a real analytic curve $T: \lambda=\lambda(\xi), \xi \in I_{\sigma_{-} / 2}$, such that $B\left(T, \epsilon_{-} / E_{-}\right) \subset M$, where $\epsilon_{-}, E_{-}$and $\sigma_{-}$are supposed to be well defined. Let $N_{+}(p)=(A+\epsilon[a]) \xi^{2 n+1}-\lambda+h_{+}(p)$, where $h_{+}=h+\widehat{h}$ with $\|\widehat{h}\|_{M} \leq \epsilon$. Suppose that $\epsilon / E \leq \epsilon_{-} / 4 E_{-}$ with $E>0$. Then there exists a domain of $\mathbb{C}^{2}, M_{+} \subset M$ with $\operatorname{dist}\left(M_{+}, \partial M\right) \geq \epsilon_{-} / 4 E_{-}$, such that $N_{+}$is real analytic on $M_{+}$ and $\widehat{h}$ satisfies $\left\|\mathscr{D}_{p} \widehat{h}\right\|_{M_{+}} \leq \epsilon E_{-} / \epsilon_{-}$. If

$$
\left\|\mathscr{D}_{p} h_{+}\right\|_{M_{+}} \leq \frac{1}{2},
$$

then, on the domain $M_{+}, N_{+}(p)=0$ determines implicitly a real analytic curve $T_{+}: \lambda=\lambda_{+}(\xi), \xi \in I_{\sigma / 2}\left(\sigma<\sigma_{-}\right)$, such that

$$
\left|\lambda_{+}(\xi)-\lambda(\xi)\right| \leq 2 \epsilon, \quad \xi \in I_{\sigma / 2},
$$

and $B\left(T_{+}, \epsilon / E\right) \subset M_{+}$. Moreover, one has

$$
\left|N_{+}(p)\right| \leq \frac{8 \epsilon}{E}, \quad \forall p \in M_{+} .
$$


Proof. Let $M_{+}=B(T, 3 \epsilon / E) \cap\left(I_{\sigma / 2+\epsilon / E} \times \mathbb{C}\right)$. Since $\epsilon / E \leq$ $\epsilon_{-} / 4 E_{-}$and $\sigma \leq \sigma_{-}$, by $B\left(T, \epsilon_{-} / E_{-}\right) \subset M$ it follows easily that $M_{+} \subset M$ and $\operatorname{dist}\left(M_{+}, \partial M\right) \geq \epsilon_{-} / 4 E_{-}$. Using Cauchy's estimate we have

$$
\left\|\mathscr{D}_{p} \widehat{h}\right\|_{M_{+}} \leq \frac{4 \epsilon E_{-}}{\epsilon_{-}} .
$$

By condition (29), the equation

$$
N_{+}(p)=(A+\epsilon[a]) \xi^{2 n+1}-\lambda+h_{+}(p)=0
$$

determines implicitly an analytic curve on $M_{+}$,

$$
T_{+}: \lambda=\lambda_{+}(\xi), \quad \xi \in I_{\sigma / 2} .
$$

It follows that

$$
\begin{aligned}
\mid \lambda_{+}(\xi) & -\lambda(\xi) \mid \\
= & \left|h\left(\xi, \lambda_{+}\right)-h(\xi, \lambda)+\widehat{h}\left(\xi, \lambda_{+}\right)\right|,
\end{aligned}
$$

so

$$
\left|\lambda_{+}(\xi)-\lambda(\xi)\right| \leq 2 \epsilon, \quad \xi \in I_{\sigma / 2} .
$$

Thus, $B\left(T_{+}, \epsilon / E\right) \subset M_{+}$. Let $E \leq 1 / 2$. For each $p=(\xi, \lambda) \epsilon$ $M_{+}$, we have

$$
\left|\lambda-\lambda_{+}(\xi)\right| \leq|\lambda-\lambda(\xi)|+\left|\lambda(\xi)-\lambda_{+}(\xi)\right| \leq \frac{3 \epsilon}{E}+2 \epsilon \leq \frac{4 \epsilon}{E} .
$$

Noting that $\left|N_{+\lambda}(p)\right| \leq 2$, for all $p \in M_{+}$, and $N_{+}(p)=0$, for all $p=\left(\xi, \lambda_{+}(\xi)\right)$, we have

$$
\left|N_{+}(p)\right| \leq \frac{8 \epsilon}{E}, \quad \forall p \in M_{+}
$$

Thus we prove Lemma 11.

Lemma 12. Assume that $R_{0}(t)=\sum_{\Lambda \in \tau} R_{0 \Lambda}(t)$ is an analytic almost-periodic function with respect to $t$ with the frequency $\omega=\left(\omega_{1}, \omega_{2}, \ldots\right)$; it has the finite spatial structure $(\tau,[\cdot])$, and

$$
\left\|R_{0}(t)\right\|_{m, s}<+\infty, \quad m>0, s>0 .
$$

If

$$
|i\langle k, \omega\rangle| \geq \frac{\alpha}{\Delta^{4}(|k|) \Delta^{4}([k])}, \quad \forall k \in \mathbb{Z}^{\infty} \backslash\{0\},
$$

then there exists an almost-periodic function $u(t)$ with the same spatial structure as $R_{0}(t)$, which satisfies

$$
\partial_{\omega} u=A \Omega u+R_{0}^{K}-\left[R_{0}\right]
$$

where $\partial_{\omega} u=\left\langle\omega, \nabla_{\theta} u\right\rangle$ is the direction derivative of $u$ along with $\omega(\theta=\omega t) . R_{0}^{K}$ is the truncation of $R_{0}$ with order $K$.

Moreover, for $0<\bar{m}<m, 0<\bar{s}<s$,

$$
\|\| u(t)\left|\left\|_{m-\bar{m}, s-\bar{s}} \leq \frac{\Gamma(\bar{m}) \Gamma(\bar{s})}{\alpha}\right\|\right| R_{0}(t)\|\|_{m, s^{*}}
$$

Proof. We assume

$$
\begin{gathered}
R_{0 \Lambda}^{K}(t)=\sum_{\operatorname{supp} k \subset \Lambda,|k| \leq K} R_{0 \Lambda k} e^{i\langle k, \omega t\rangle}, \\
u_{\Lambda}(t)=\sum_{\operatorname{supp} k \subset \Lambda} u_{\Lambda k} e^{i\langle k, \omega t\rangle} .
\end{gathered}
$$

Insert the above formulas into the equation $\partial_{\omega} u=A \Omega u+$ $R_{0}^{K}-\left[R_{0}\right]$, and compare the coefficients on both sides, thus we can find

$$
u_{\Lambda k}=\frac{R_{0 \Lambda k}}{i\langle k, \omega\rangle-A \Omega}, \quad 0<|k| \leq K, \quad \operatorname{supp} k \subset \Lambda .
$$

Then

$$
\begin{aligned}
& \left\|u_{\Lambda}(t)\right\|_{\mathbb{T}_{s-\bar{s}}} \\
& \leq \sum_{\operatorname{supp} k \subset \Lambda, 0 \leq|k| \leq K} \frac{\Delta^{4}(|k|) e^{-\bar{s}|k|}}{\alpha} \Delta^{4}([k])\left|R_{0 \Lambda k}\right| e^{s|k|} \\
& \leq \frac{\Gamma(\bar{s}) \Delta^{4}([\Lambda])}{\alpha}\left\|R_{0 \Lambda}\right\|_{\mathbb{T}_{s}} .
\end{aligned}
$$

From Definition 7,

$$
\begin{aligned}
& \|u(t) \mid\| \|_{m-\bar{m}, s-\bar{s}} \\
& =\sum_{\Lambda \in \tau}\left\|u_{\Lambda}\right\|_{\mathbb{T}_{s}-\bar{s}} e^{(m-\bar{m})[\Lambda]} \\
& \leq \sum_{\Lambda \in \tau} \frac{\Gamma(\bar{s}) \Delta^{4}([\Lambda])}{\alpha}\left\|R_{0 \Lambda}\right\|_{\mathbb{T}_{s}} \\
& \leq \frac{\Gamma(\bar{m}) \Gamma(\bar{s})}{\alpha} \sum_{\Lambda \in \tau}\left\|R_{0 \Lambda}\right\|_{\mathbb{T}_{s}} e^{m[\Lambda]} \\
& =\frac{\Gamma(\bar{m}) \Gamma(\bar{s})}{\alpha}\left\|R_{0}(t)\right\| \|_{m, s} .
\end{aligned}
$$

Thus, $u(t)=\sum_{\Lambda \in \tau} u_{\Lambda}(t)$ is convergent in the smaller domain $D_{s-\bar{s}}$ with the norm ||$\cdot|| \|_{m-\bar{m}, s-\bar{s}}$.

Now we consider the following real analytic almostperiodic differential equation with parameters

$$
\dot{x}=N(p)+A \Omega(p) x+\epsilon S(t, p) x+P(x, t, p)
$$

with $S(t, p)=a(t) \Omega(p)$

Lemma 13. Consider the above equation, where $N(p)=(A+$ $\epsilon[a]) \xi^{2 n+1}-\lambda+h(p)$. Let $\widehat{h}=\left[\left.P\right|_{x=0}\right]$ and $\widehat{\Omega}=\left(\left.P_{x}\right|_{x=0}\right)^{K}$. Let $h_{+}=h+\widehat{h}, \Omega_{+}=A \Omega+\widehat{\Omega}$ and $N_{+}(p)=(A+\epsilon[a]) \xi^{2 n+1}-\lambda+$ $h_{+}(p)$. Assume the following hold.

(1) $P$ is real analytic on $\Delta_{r, s} \times M$ and satisfies

$$
\||| P\|_{m, \Delta_{r, s} \times M} \leq \epsilon=\frac{\alpha r E}{\Gamma(m / 2) \Gamma(\rho)}
$$

with $0 \leq E<1 / 2,0<\rho<s / 2$, and $E=e^{-\rho K}$. 
(2) $4 \epsilon / E \leq \sigma$.

(3) $N, \widehat{h}$, and $h_{+}$are real analytic on $M \subset C^{2}$ and all the assumptions of Lemma 11 hold. Let $M_{+} \subset M$ be the domain defined in Lemma 11.

Then, for any $p \in M_{+}$, there exists an affine analytic almost-periodic transformation

$$
\begin{aligned}
& \Phi(\cdot, t ; p): x_{+} \in D\left(0, r_{+}\right) \longrightarrow x \\
& =(1+\epsilon Q) x_{+}+u(t ; p) \in D(0, r),
\end{aligned}
$$

where $\Phi$ is real analytic on $D\left(r_{+}, s_{+}\right) \times M_{+}$, such that the above differential equation is transformed to

$$
\dot{x}_{+}=N_{+}(p)+\Omega_{+}(p) x_{+}+\epsilon^{2} S_{+}(t, p) x_{+}+P_{+}\left(x_{+}, t, p\right),
$$

where $S_{+}$and $P_{+}$will be get in the proof.

Moreover, one has

$$
\begin{gathered}
\|\mid \Phi-i d\| \|_{m, \Delta_{r, s-2 \rho} \times M_{+}} \leq c\left(r+r^{2}\right) E, \\
\left\|\mid D_{x} \Phi-1\right\|_{m, \Delta_{r, s-2 \rho} \times M_{+}} \leq c r E .
\end{gathered}
$$

The new perturbation term $P_{+}$satisfies

$$
\left\|P_{+}\right\| \|_{m_{+}, \Delta_{r_{+}, s_{+}} \times M_{+}} \leq \epsilon_{+}=\frac{\alpha r_{+} E_{+}}{\Gamma\left(m_{+} / 2\right) \Gamma\left(\rho_{+}\right)}
$$

with

$$
\begin{aligned}
& s_{+}=s-2 \rho, \quad \eta=E^{1 / 2}, \\
& \rho_{+}=\frac{1}{2} \rho, \quad r_{+}=\eta \rho, \\
& E_{+}=c E^{3 / 2}, \quad m_{+}=\frac{1}{2} m .
\end{aligned}
$$

Proof. The proof is the standard KAM step and we divide it into several parts.

(A) Truncation. Let $R=R_{0}+R_{1} x$ with $R_{0}=\left.P\right|_{x=0}$ and $R_{1}=$ $\left.P_{x}\right|_{x=0}$. It follows easily that

$$
\left\|R_{0}\right\|_{m, \Delta_{r, s} \times M} \leq \epsilon, \quad\|\| R_{1} \|_{m, \Delta_{r, s} \times M} \leq \frac{\epsilon}{r} .
$$

Hence $\||| R \mid\|_{m, \Delta_{r, s} \times M} \leq 2 \epsilon$. Let

$$
\begin{aligned}
& R=\sum_{\Lambda \in \tau, \operatorname{supp} k \subset \Lambda, k \in Z^{\infty}} R_{k}(x ; p) e^{i\langle k, \omega t\rangle}, \\
& R^{K}=\sum_{\Lambda \in \tau, \operatorname{supp} k \subset \Lambda,|k| \leq K} R_{k}(x ; p) e^{i\langle k, \omega t\rangle} .
\end{aligned}
$$

By definition, we have

$$
\left\|R-R^{K}\right\|_{m, \Delta_{r, s-\rho} \times M} \leq 2 \epsilon e^{-K \rho} .
$$

(B) Construnction of the Transformation. Define the transformation $\phi_{1}: x=u(t)+y$, where $u$ satisfies

$$
\partial_{\omega} u=A \Omega u+R_{0}^{K}-\left[R_{0}\right] .
$$

From Lemma 12, we have

$$
\begin{aligned}
\|u(t)\| \|_{m-(m / 2), s-2 \rho} & \\
\leq & \frac{\Gamma(m / 2) \Gamma(2 \rho)}{\alpha}\left\|R_{0}(t)\right\|_{m, s} \\
& \leq \frac{\Gamma(m / 2) \Gamma(2 \rho)}{\alpha} \epsilon<c r E .
\end{aligned}
$$

By the transformation $\phi_{1}$, the equation becomes

$$
\begin{aligned}
\dot{u}+\dot{y}= & N(p)+A \Omega(p)(u+y) \\
& +\epsilon a(t) \Omega(p)(u+y)+P(u+y, t, p), \\
\dot{y}= & N(p)+\left[R_{0}\right]+\left(A \Omega(p)+R_{1}^{K}\right) y+\epsilon a(t) \Omega(p) y \\
& +R(u+y, t)-R^{K}(u+y, t)+R_{1}^{K} u \\
& +\epsilon a(t) \Omega(p) u+P(u+y, t)-R(u+y, t) ;
\end{aligned}
$$

then

$$
\begin{aligned}
\dot{y}= & N_{+}(p)+\left(A \Omega(p)+R_{1}^{K}\right) y+\epsilon a(t) \Omega(p) y \\
& +R(u+y, t)-R^{K}(u+y, t)+R_{1}^{K} u \\
& +\epsilon a(t) \Omega(p) u+P(u+y, t)-R(u+y, t) .
\end{aligned}
$$

Define the transformation $\phi_{2}: y=(1+\epsilon Q) x_{+} ; Q$ satisfies

$$
\dot{Q}=a(t) \Omega(p) \text {. }
$$

We assume

$$
\begin{array}{rlrl}
Q(t) & =\sum_{\Lambda \in \tau} Q_{\Lambda}(t), & Q_{\Lambda}(t)=\sum_{\operatorname{supp} k \subset \Lambda,} Q_{\Lambda k} e^{i\langle k, \omega t\rangle}, \\
a(t)=\sum_{\Lambda \in \tau} a_{\Lambda}(t), & a_{\Lambda}(t)=\sum_{\operatorname{supp} k \subset \Lambda,} a_{\Lambda k} e^{i\langle k, \omega t\rangle} .
\end{array}
$$

Then

$$
Q_{\Lambda k}= \begin{cases}0, & |k|=0, \operatorname{supp} k \subset \Lambda, \\ \frac{a_{\Lambda k} \Omega}{i\langle k, \omega\rangle}, & |k|>0, \operatorname{supp} k \subset \Lambda .\end{cases}
$$

Similar to Lemma 12, we have

$$
\begin{aligned}
& \left\||Q(t) \||_{m-(m / 2), s-2 \rho}\right. \\
& \quad \leq \frac{\Gamma(m / 2) \Gamma(2 \rho)}{\alpha}\left|\|a(t)\| \|_{m, s}\right| \Omega \mid \\
& \leq c \frac{\Gamma(m / 2) \Gamma(2 \rho)}{\alpha}, \\
& \left.\|\in Q(t)\|\right|_{m-(m / 2), s-2 \rho} \leq c r E .
\end{aligned}
$$


By the transformation $\phi_{2}$, the equation becomes

$$
\begin{aligned}
&(1+\epsilon Q) \dot{x}_{+}+\epsilon \dot{Q} x_{+} \\
&=N_{+}(p)+\left(A \Omega(p)+R_{1}^{K}\right)(1+\epsilon Q) x_{+} \\
&+\epsilon a(t) \Omega(p)(1+\epsilon Q) x_{+} \\
&+R\left(u+(1+\epsilon Q) x_{+}, t\right)-R^{K}\left(u+(1+\epsilon Q) x_{+}, t\right) \\
&+R_{1}^{K} u+\epsilon a(t) \Omega(p) u \\
&+P\left(u+(1+\epsilon Q) x_{+}, t\right)-R\left(u+(1+\epsilon Q) x_{+}, t\right)
\end{aligned}
$$

then

$$
\begin{aligned}
\dot{x}_{+}= & N_{+}(p)+\left(A \Omega(p)+R_{1}^{K}\right) x_{+}+\epsilon^{2} \frac{a(t) \Omega(p) Q}{1+\epsilon Q} x_{+} \\
& +\left(\frac{1}{1+\epsilon Q}-1\right) N_{+}+\frac{1}{1+\epsilon Q} R\left(u+(1+\epsilon Q) x_{+}, t\right) \\
& -\frac{1}{1+\epsilon Q} R^{K}\left(u+(1+\epsilon Q) x_{+}, t\right) \\
& +\frac{1}{1+\epsilon Q} R_{1}^{K} u+\frac{\epsilon a(t) \Omega(p) u}{1+\epsilon Q} \\
& +\frac{1}{1+\epsilon Q} P\left(u+(1+\epsilon Q) x_{+}, t\right) \\
& -\frac{1}{1+\epsilon Q} R\left(u+(1+\epsilon Q) x_{+}, t\right) .
\end{aligned}
$$

Thus, by the transformation $\Phi=\phi_{1} \circ \phi_{2}: x=u+(1+$ $\epsilon Q) x_{+}$, the equation is transformed to

$$
\dot{x}_{+}=N_{+}+\Omega_{+} x_{+}+\epsilon^{2} S_{+} x_{+}+P_{+}\left(x_{+}, t ; p\right) \text {, }
$$

where

$$
\begin{aligned}
& P_{+}=\left(\frac{1}{1+\epsilon Q}-1\right) N_{+}+\frac{1}{1+\epsilon Q} R\left(u+(1+\epsilon Q) x_{+}, t\right) \\
&-\frac{1}{1+\epsilon Q} R^{K}\left(u+(1+\epsilon Q) x_{+}, t\right) \\
&+\frac{1}{1+\epsilon Q} R_{1}^{K} u+\frac{\epsilon a(t) \Omega(p) u}{1+\epsilon Q} \\
&+\frac{1}{1+\epsilon Q} P\left(u+(1+\epsilon Q) x_{+}, t\right) \\
&-\frac{1}{1+\epsilon Q} R\left(u+(1+\epsilon Q) x_{+}, t\right) \\
& S_{+}=\frac{a(t) \Omega(p) Q}{1+\epsilon Q} .
\end{aligned}
$$

With the estimates of $u$ and $Q$, we have

$$
\begin{gathered}
\|\| \Phi-i d\|\|_{m, \Delta_{r, s-2 \rho} \times M_{+}} \\
\leq r\|\epsilon Q\|\left\|_{m, \Delta_{r, s-2 \rho} \times M_{+}}+\right\|\|u\|_{m, \Delta_{r, s-2 \rho} \times M_{+}} \\
\leq c\left(r+r^{2}\right) E, \\
\left\|D_{x} \Phi-1\left|\left\|_{m, \Delta_{r, s-2 \rho} \times M_{+}} \leq\right\|\right| \epsilon Q\right\| \|_{m, \Delta_{r, s-2 \rho} \times M_{+}} \leq c r E .
\end{gathered}
$$

Let $\eta=E^{1 / 2} \leq 1 / 4, s_{+}=s-2 \rho$, and $r_{+}=\eta r$. Then the transformation $\Phi: x_{+} \in D\left(0, r_{+}\right) \rightarrow D\left(0,2 r_{+}\right)$is analytic almost-periodic on $\mathbb{T}_{s_{+}}$with respect to $t$ and affine in $x_{+}$.

(C) Estimates of Error Terms. Because $\|\mid \epsilon Q\| \|_{m_{+}, \Delta_{r_{+}, s_{+}} \times M_{+}}<1$, then $1 /(1+\epsilon Q)=1-\epsilon Q+(\epsilon Q)^{2}-(\epsilon Q)^{3}+\cdots+(-\epsilon Q)^{n}+\cdots$. Thus,

$$
\begin{gathered}
\|\| \frac{1}{1+\epsilon Q}\|\|_{m_{+}, \Delta_{r_{+}, s_{+}} \times M_{+}} \\
\leq 1+\|\epsilon Q\|\left\|_{m_{+}, \Delta_{r_{+}, s_{+}} \times M_{+}}+\right\| \mid \epsilon Q\|\|_{m_{+}, \Delta_{r_{+}, s_{+}} \times M_{+}}^{2} \\
+\cdots+\|\mid\| Q\|\|_{m_{+}, \Delta_{r_{+}, s_{+}} \times M_{+}}^{n}+\cdots
\end{gathered}
$$

$\leq 2$

Let $\eta=E^{1 / 2}$. Then, it follows that

$$
\begin{aligned}
& \left\|\left(\frac{1}{1+\epsilon Q}-1\right) N_{+}\right\| \|_{m_{+}, \Delta_{r_{+}, s_{+}} \times M_{+}} \\
& \leq\left\|\left|\epsilon Q N_{+}\right|\right\|_{m_{+}, \Delta_{r_{+}, s_{+}} \times M_{+}} \leq c \epsilon E, \\
& \| \frac{1}{1+\epsilon Q}\left(R \circ \Phi-R^{K} \circ \Phi\right)||_{m_{+}, \Delta_{r_{+}, s_{+}} \times M_{+}} \leq c \epsilon e^{-K \rho}, \\
& \left\|\frac{1}{1+\epsilon Q} R_{1}^{K} u\right\| \|_{m_{+}, \Delta_{r_{+}, s_{+}} \times M_{+}} \leq 2 \frac{\epsilon}{r} c r E \leq c \epsilon E, \\
& \left.\left\|\frac{\epsilon a(t) \Omega u}{1+\epsilon Q}\right\|\right|_{m_{+}, \Delta_{r_{+}, s_{+}} \times M_{+}} \leq c \epsilon E, \\
& \left\|\frac{1}{1+\epsilon Q}(P \circ \Phi-R \circ \Phi) \mid\right\|_{m_{+}, \Delta_{r_{+}, s_{+}} \times M_{+}} \\
& \leq c \frac{\epsilon}{r^{2}} r^{2} \eta^{2}=c \eta^{2} \epsilon \text {. }
\end{aligned}
$$

So

$$
\begin{aligned}
& \left\|P_{+}\right\|_{m_{+}, \Delta_{r_{+}, s_{+}} \times M_{+}} \\
& \quad \leq c \epsilon E+c \epsilon e^{-K \rho}+c \eta^{2} \epsilon=c \epsilon E \\
& \quad=\frac{\alpha r_{+} E_{+}}{\Gamma\left(m_{+} / 2\right) \Gamma\left(\rho_{+}\right)}=\epsilon_{+}
\end{aligned}
$$


where $\rho_{+}=(1 / 2) \rho, r_{+}=\eta r, E_{+}=c E^{3 / 2}$, and $m_{+}=(1 / 2) m$. Thus we have proved Lemma 13.

Iteration. Now we choose some suitable parameters so that the above KAM step can be iterated infinitely. At the initial step, let

$$
\begin{aligned}
& s_{0}=s, \quad r_{0}=r, \quad E_{0}>0, \quad \eta_{0}=E_{0}^{1 / 2}, \\
& \rho_{0}=\frac{s_{0}}{8}, \quad \epsilon_{0}=\frac{\alpha r_{0} E_{0}}{\Gamma\left(m_{0} / 2\right) \Gamma\left(\rho_{0}\right)}, \quad m_{0}=\frac{s_{0}}{8} .
\end{aligned}
$$

Let $K_{0}$ satisfy $E_{0}=e^{-K_{0} \rho_{0}}$ and $\sigma_{0}=\delta$. Inductively, we define

$$
\begin{gathered}
s_{j}=s_{j-1}-2 \rho_{j-1}, \quad \eta_{j}=E_{j}^{1 / 2}, \quad \rho_{j}=\frac{1}{2} \rho_{j-1}, \\
r_{j}=\eta_{j-1} r_{j-1}, \quad E_{j}=c E_{j-1}^{3 / 2}, \quad m_{j}=\frac{1}{2} m_{j-1}, \\
\epsilon_{j}=\frac{\alpha r_{j} E_{j}}{\Gamma\left(m_{j} / 2\right) \Gamma\left(\rho_{j}\right)}, \quad \sigma_{j}=\frac{\sigma_{j-1}}{4} .
\end{gathered}
$$

And $K_{j}$ satisfies $E_{j}=e^{-K_{j} \rho_{j}}$.

By $E_{j}=c E_{j-1}^{3 / 2}$, we have $E_{j}=c^{-2}\left(c^{2} E_{0}\right)^{(3 / 2)^{j}}$. Thus, if $E_{0}$ is sufficiently small, we have $c E_{j} \leq 1$ and $\eta_{j}=E_{j}^{1 / 2} \leq 1 / 4$. Moreover, by definition it follows that

$$
\frac{\left(\epsilon_{j+1} / E_{j+1}\right)}{\left(\epsilon_{j} / E_{j}\right)}=\frac{\Gamma\left(m_{j} / 2\right) \Gamma\left(\rho_{j}\right)}{\Gamma\left(m_{j+1} / 2\right) \Gamma\left(\rho_{j+1}\right)} E_{j}^{1 / 2} \leq E_{j}^{1 / 2} \leq \frac{1}{4} \leq 1 .
$$

Thus $\epsilon_{j+1} / E_{j+1} \leq \epsilon_{j} / E_{j}, j \leq 0$.

Now we prove that, for $E_{0}$ sufficiently small, $4 \epsilon_{j} / E_{j} \leq \sigma_{j}$ hold for all $j \leq 0$.

Let $G_{j}=4 \epsilon_{j} / E_{j} \sigma_{j}$; from (21) we have $G_{0} \leq 1$. Moreover, we have

$$
\begin{aligned}
\frac{G_{j+1}}{G_{j}} & =\frac{\sigma_{j} \epsilon_{j+1} E_{j}}{\sigma_{j+1} \epsilon_{j} E_{j+1}} \\
& =4 \frac{\alpha r_{j+1} E_{j+1}}{\Gamma\left(m_{j+1} / 2\right) \Gamma\left(\rho_{j+1}\right)} \cdot \frac{\Gamma\left(m_{j} / 2\right) \Gamma\left(\rho_{j}\right)}{\alpha r_{j} E_{j}} \cdot \frac{E_{j}}{E_{j+1}} \\
& \leq 4 \frac{\eta_{j} E_{j+1}}{E_{j}} \cdot \frac{E_{j}}{E_{j+1}}=4 E_{j}^{1 / 2} \leq 1,
\end{aligned}
$$

for all $j \leq 0$. Thus, $G_{j} \leq G_{0} \leq 1$. So the inequalities in the assumption 2 of Lemma 13 hold for all $j \leq 0$.

Let $M_{0}=M, N_{0}=(A+\epsilon[a]) \xi^{2 n+1}-\lambda, h_{0}=0, \Omega_{0}=$ $A \Omega, S_{0}=a \Omega$, and $P_{0}=P$. By Lemmas 11,12 , and 13, we have a sequence of closed domains $M_{j}$ with $M_{j+1} \subset M_{j}$ and a sequence of affine transformations

$$
\begin{gathered}
\Phi_{j}: D\left(0, r_{j+1}\right) \longrightarrow D\left(0,2 r_{j+1}\right) \subset D\left(0, r_{j}\right), \\
\Phi_{j}: x_{j}=\left(1+\epsilon^{2^{j}} Q_{j}\right) x_{j+1}+u_{j} .
\end{gathered}
$$

We also have

$$
\begin{gathered}
\left\|\left|\Phi_{j}-i d\right|\right\|_{m_{j+1}, \Delta_{r_{j+1}, s_{j+1}} \times M_{j+1}} \leq c\left(r_{j}+r_{j}^{2}\right) E_{j}, \\
\left\|\left|D_{x} \Phi_{j}-1 \|\right|_{m_{j+1}, \Delta_{r_{j+1}, s_{j+1}} \times M_{j+1}} \leq c r_{j} E_{j} .\right.
\end{gathered}
$$

Let $\Phi^{j}=\Phi_{0} \circ \Phi_{1} \circ \cdots \Phi_{j-1}$ with $\Phi_{0}=i d$. Then, after the transformation $\Phi^{j},(19)$ is changed to

$$
\dot{x}=N_{j}(p)+\Omega_{j}(p) x+\epsilon^{2^{j}} S_{j} x+P_{j}(x, t ; p) .
$$

By the inductive assumptions of KAM iteration, we have $\left.\left\|P_{j}\right\|\right|_{m_{j}, \Delta_{r_{j}, s_{j}} \times M_{j}} \leq \epsilon_{j}$.

The correction terms $\widehat{h}_{j}$ and $\widehat{\Omega}_{j}$ satisfy

$$
\left\|\widehat{h}_{j}\right\|_{M_{j}} \leq \epsilon_{j}, \quad\left\|\widehat{\Omega}_{j}\right\|_{M_{j}} \leq \frac{\epsilon_{j}}{r_{j}} .
$$

By Lemma 11, we have $\operatorname{dist}\left(M_{j+1}, \partial M_{j}\right) \geq \epsilon_{j-1} / 4 E_{j-1}$. For Cauchy's estimate we have

$$
\left\|D_{p} \widehat{h}_{j}\right\|_{M_{j+1}} \leq \frac{4 \epsilon_{j} E_{j-1}}{\epsilon_{j-1}} .
$$

Noting that $M_{0}=B\left(I \times J, \sigma_{0}\right)$ and $T_{0}: \lambda=(A+\epsilon[a]) \xi^{2 n+1}$, $|\xi| \leq \delta \leq 1$. Since $\sigma_{0} \geq 4 \epsilon_{0} / E_{0}$ and $M_{1}=B\left(T_{0}, 3 \epsilon_{0} / E_{0}\right) \cap$ $\left(I_{\sigma_{0} / 2+\epsilon_{0} / E_{0}} \times \mathbb{C}\right)$, it follows that $M_{1} \subset M_{0}$ and $\operatorname{dist}\left(M_{1}, \partial M_{0}\right) \geq$ $\sigma_{0} / 4$. For Cauchy's estimate we have

$$
\left\|D_{p} \widehat{h}_{0}\right\|_{M_{1}} \leq \frac{4 \epsilon_{0}}{\sigma_{0}} .
$$

Let $F_{j}=\epsilon_{j} E_{j-1} / \epsilon_{j-1} r_{j}$; then

$$
\begin{aligned}
F_{j} & =\frac{E_{j-1}}{r_{j}} \cdot \frac{\alpha r_{j} E_{j} \Gamma\left(m_{j-1} / 2\right) \Gamma\left(\rho_{j-1}\right)}{\alpha r_{j-1} E_{j-1} \Gamma\left(m_{j} / 2\right) \Gamma\left(\rho_{j}\right)} \leq \frac{E_{j-1}}{r_{j}} \cdot \frac{\alpha r_{j} E_{j}}{\alpha r_{j-1} E_{j-1}} \\
& =\frac{c E_{j-1}^{2}}{r_{j}}=R_{j} .
\end{aligned}
$$

So $R_{j+1} / R_{j}=c E_{j}^{1 / 6}$. Obviously, we can choose $E_{0}$ sufficiently small so that $R_{j+1} / R_{j} \leq 1 / 2, R_{j+1} \leq(1 / 2) R_{j}$. Noting that $h_{0}=0$ and $\epsilon_{0} / r_{0} \sigma_{0} \leq 1 / 16$, we have

$$
\begin{aligned}
\left\|D_{p} h_{j}\right\|_{M_{j+1}} & \leq\left\|D_{p} \widehat{h}_{0}\right\|_{M_{j+1}}+\sum_{l=1}^{j-1}\left\|D_{p} \widehat{h}_{l}\right\|_{M_{j+1}} \\
& \leq \frac{4 \epsilon_{0}}{\sigma_{0}}+\sum_{l=1}^{j-1} 4 r_{j} R_{j} \leq \frac{1}{4}+c E_{0}^{2} \leq \frac{1}{2} .
\end{aligned}
$$

So condition (29) holds for all $j \leq 0$.

From Lemma 11, $N_{j}(p)=(A+\epsilon[a]) \xi^{2 n+1}-\lambda+h_{j}(p)=0$ defines implicitly a real analytic curve $T_{j} \subset M_{j}: \lambda=\lambda_{j}(\xi)$, $\xi \in I_{\sigma_{j} / 2}$, satisfying

$$
\left|\lambda_{j+1}(\xi)-\lambda_{j}(\xi)\right| \leq 2 \epsilon_{j}, \quad \forall \xi \in I_{\sigma_{j} / 2} .
$$

Furthermore, $\left\|N_{j+1}\right\|_{M_{j+1}} \leq 8 \epsilon_{j} / E_{j}$. 
Convergence of KAM Iteration. Now we prove the convergence of KAM iteration. By the definition of $E_{j}$, if $E_{0}$ is sufficiently small, it follows that

$$
\left\|D_{x} \Phi_{j} \mid\right\|_{m_{j}, \Delta_{r_{j}, s_{j}} \times M_{j}} \leq \prod_{i=0}^{j-1}\left(1+c r_{i} E_{i}\right) \leq 2 .
$$

Therefore, we have

$$
\begin{gathered}
\|\| \Phi^{j}-\Phi^{j-1} \|\left.\right|_{m_{j}, \Delta_{r_{j}, s_{j}} \times M_{j}} \leq c\left(r_{j-1}+r_{j-1}^{2}\right) E_{j-1}, \\
\left\|D_{x}\left(\Phi^{j}-\Phi^{j-1}\right)\right\|_{m_{j}, \Delta_{r_{j}, s_{j}} \times M_{j}} \leq c r_{j-1} E_{j-1} .
\end{gathered}
$$

Let

$$
\begin{aligned}
& \Delta_{*}=\bigcap_{j=0}^{\infty} \Delta_{r_{j}, s_{j}}=\Delta_{0, s_{0} / 2}, \quad M_{*}=\bigcap_{j \geq 0} M_{j}, \\
& \Phi=\lim _{j \rightarrow \infty} \Phi^{j} .
\end{aligned}
$$

We have

$$
\begin{aligned}
\left\|\Phi^{j}-\Phi^{0}\right\| & \|_{m_{j}, \Delta_{r_{j}, s_{j}} \times M_{j}} \\
\leq & \left\|\Phi^{j}-\Phi^{j-1}\right\| \|_{m_{j}, \Delta_{r_{j}, s_{j}} \times M_{j}} \\
& +\|\| \Phi^{j-1}-\Phi^{j-2} \mid \|_{m_{j}, \Delta_{r_{j}, s_{j}} \times M_{j}} \\
& +\cdots+\|\| \Phi^{1}-\Phi^{0} \|_{m_{j}, \Delta_{r_{j}, s_{j}} \times M_{j}} \\
\leq & c\left(r_{j-1}+r_{j-1}^{2}\right) E_{j-1}+c\left(r_{j-2}+r_{j-2}^{2}\right) E_{j-2} \\
& +\cdots+c\left(r_{0}+r_{0}^{2}\right) E_{0} \\
\leq & c\left(r_{0}+r_{0}^{2}\right) E_{0} .
\end{aligned}
$$

Thus

$$
\begin{gathered}
\||\Phi-i d|\|_{m_{*}, \Delta_{r_{*}, s_{*}} \times M_{*}} \leq c\left(r_{0}+r_{0}^{2}\right) E_{0} \\
\||| D_{x} \Phi-i d||_{m_{*}, \Delta_{r_{*}, s_{*}} \times M_{*}} \leq c r_{0} E_{0}
\end{gathered}
$$

So we have the convergence of $\Phi^{j}$ to $\Phi$ on $\Delta_{r_{0} / 2, s_{0} / 2}$.

From (86) it is easy to show that $\lambda_{j}$ is convergent on $I$. In fact, $\epsilon_{j+1} / \epsilon_{j} \leq c E_{j} \leq 1 / 2$. For $i>j$, it follows that

$$
\left|\lambda_{i}(\xi)-\lambda_{j}(\xi)\right| \leq \sum_{l=j}^{i-1} 2 \epsilon_{l} \leq 4 \epsilon_{j}, \quad \forall \xi \in I_{(1 / 2) \sigma_{j}} .
$$

Let $\lambda_{i}(\xi) \rightarrow \lambda(\xi)=(A+\epsilon[a]) \xi^{2 n+1}+\widehat{N}(\xi), \xi \in I$. For $\lambda_{0}(\xi)=$ $(A+\epsilon[a]) \xi^{2 n+1}$, we have

$$
\begin{gathered}
\left|\lambda_{i}(\xi)-(A+\epsilon[a]) \xi^{2 n+1}\right| \leq 4 \epsilon_{0}, \quad \forall \xi \in I_{(1 / 2) \sigma_{j}}, \\
|\widehat{N}(\xi)|=\left|\lambda(\xi)-(A+\epsilon[a]) \xi^{2 n+1}\right| \leq 4 \epsilon_{0}, \quad \forall \xi \in I .
\end{gathered}
$$

Moreover, by Cauchy's estimate we have

$$
\left|\lambda_{j+1}^{\prime}(\xi)-\lambda_{j}^{\prime}(\xi)\right| \leq \frac{4 \epsilon_{j}}{\sigma_{j}}, \quad \forall \xi \in I .
$$

Let $L_{j}=4 \epsilon_{j} / \sigma_{j}$; then $L_{j+1} / L_{j} \leq c E_{j}$. Thus, it is easy to prove that $\left\{\lambda_{j}^{\prime}(\xi)\right\}$ is convergent uniformly on $I$, and so $\lambda(\xi)$ is differentiable on $I$. In fact, in the same way as in [7], we can prove that $\lambda(\xi)$ is $C^{\infty}$-smooth on $I$.

Since $T_{i} \subset M_{i} \subset M_{j}$, for all $i \geq j$, letting $i \rightarrow \infty$ we have $T=\left\{(\xi, \lambda) \mid \lambda=\lambda(\xi), \xi \in I \subset M_{j}\right\}$ and $T=M_{*}=\cap_{j \leq 0} M_{j}$. Obviously, $N_{j}(p) \rightarrow 0$, for $p \in T$. Let $\Omega_{j} \rightarrow \Omega_{*}$ and let $P_{j} \rightarrow P_{*}$. By Cauchy's estimate we have

$$
\left|D_{x} P_{j}\right|_{x=0} \mid \leq \frac{\epsilon_{j}}{r_{j}}=\frac{\alpha E_{j}}{\Gamma\left(m_{j} / 2\right) \Gamma\left(\rho_{j}\right)} \longrightarrow 0 .
$$

Thus $\left.P_{*}\right|_{x=0}=0$ and $\left.D_{x} P_{*}\right|_{x=0}=0$. Hence $P_{*}(x, t ; p)=O\left(x^{2}\right)$ for $p \in T$.

Noting that $\left\|\widehat{\Omega}_{j}\right\|_{M_{j}} \leq \epsilon_{j} / r_{j}$ and $\Omega_{j}=A \Omega+\sum_{i=1}^{j-1} \widehat{\Omega}_{j}$, we have

$$
\left|\Omega_{*}(p)-A \Omega(p)\right| \leq \frac{2 \epsilon_{0}}{r_{0}}, \quad \forall p \in \Gamma .
$$

The proof of Theorem 10 is complete.

\section{Proof of Theorem 9}

Let $x=\xi+y$ with $|\xi| \leq \delta$ and $|y| \leq r_{0} \leq \delta$. Then (5) becomes

$$
\dot{y}=(A+\epsilon a(t))(y+\xi)^{2 n+1}+h(y+\xi, t)+f(y+\xi, t) .
$$

Let

$$
(y+\xi)^{2 n+1}=\xi^{2 n+1}+(2 n+1) \xi^{2 n} y+g(y, \xi)
$$

with

$$
g(y, \xi)=\frac{(2 n+1) 2 n}{2} \xi^{2 n-1} y^{2}+\cdots+(2 n+1) \xi y^{2 n}+y^{2 n+1} .
$$

We write $h(y+\xi, t)=h(y, t, \xi)$ and $f(y+\xi, t)=f(y, t, \xi)$ and decompose $a(t)$ as $a(t)=[a]+\widetilde{a(t)}$, where $[a]$ is the average of $a(t)$ and $\widetilde{a(t)}$ has zero mean value. Then the differential equation (5) becomes

$$
\begin{aligned}
\dot{y}= & (A+\epsilon[a]) \xi^{2 n+1}+(2 n+1)(A+\epsilon a(t)) \xi^{2 n} y \\
& +P(y, t, \xi),
\end{aligned}
$$

where $P=\epsilon \widetilde{a(t)} \xi^{2 n+1}+(A+\epsilon a(t)) g+h+f$.

Let $I=[-\delta, \delta]$ and $I_{\sigma}=\{\xi \in \mathbb{C} \mid \operatorname{dist}(\xi, I) \leq \sigma\}$. By assumption and the choice of $r$ and $\delta$, it is easy to see that $g$, $h$, and $f$ are all real analytic on $\Delta_{r_{0}, s_{0}} \times I_{\sigma}$ with $r_{0} \leq \delta, \sigma_{0} \leq \delta$ and $s_{0}=s$. Moreover, we have that

$$
\begin{gathered}
\|\mid(A+\epsilon a(t)) g\|_{m_{0}, \Delta_{r_{0}, s_{0}} \times I_{\sigma_{0}}} \leq c r_{0}^{2} \delta, \\
\left\|\left.\left|\epsilon \widetilde{a(t)} \xi^{2 n+1} \|_{m_{0}, \Delta_{r_{0}, s_{0}} \times I_{\sigma_{0}}} \leq c \epsilon\right| \delta\right|^{2 n+1} \leq c \epsilon,\right.
\end{gathered}
$$


where $c$ is a constant depending on $n$. Note that we always use $c$ to denote different constants in estimates. Similarly, we have $\||h|\|_{m_{0}, \Delta_{r_{0}, s_{0}} \times I_{\sigma_{0}}} \leq c \delta^{2 n+2}$ and \|\|$f\|\|_{m_{0}, \Delta_{r_{0}, s_{0}} \times I_{\sigma_{0}}} \leq \epsilon$. Let $\delta=\epsilon^{1 /(2 n+2)}$ and let $r_{0}=\epsilon^{2 n+1 /(4 n+4)}$. Then $\delta^{2 n+2}=\epsilon$ and $r_{0}=\delta^{2 n+1 / 2}$. Let $\sigma_{0}=\delta$. Then it follows that

$$
\||P|\|_{m_{0}, \Delta_{r_{0}, s_{0}} \times I_{\sigma_{0}}} \leq c r_{0}^{2} \delta+c \delta^{2 n+2}+\epsilon=c \epsilon=\epsilon_{0} .
$$

Now (97) is equivalent to the following parameterized differential equation:

$$
\dot{y}=(A+\epsilon[a]) \xi^{2 n+1}+(A+\epsilon a(t)) \Omega(\xi) y+P(y, t, \xi),
$$

where $\Omega(\xi)=(2 n+1) \xi^{2 n}$.

Now we want to prove that if $\epsilon$ is sufficiently small, then there exists $\xi_{*} \in I$ such that at $\xi=\xi_{*}$ the differential equation (103) is reducible to a normal form with zero as equilibrium point. We introduce an external parameter and consider the following almost-periodic differential equation:

$$
\dot{x}=(A+\epsilon[a]) \xi^{2 n+1}-\lambda+(A+\epsilon a(t)) \Omega(\xi) y+P(y, t, \xi),
$$

where $\lambda \in J=[-1,1]$ is an external parameter. Obviously, (103) corresponds to (104) with $\lambda=0$.

By Theorem 10, we will prove that there exists a smooth curve $T: \lambda=\lambda(\xi), \xi \in I$, such that for $(\xi, \lambda) \in T$ the differential equation (104) can be reduced to a normal form with zero equilibrium. Moreover, we can find $\xi_{*} \in I$ such that $\lambda\left(\xi_{*}\right)=0$ and then come back to the original equation (103) with $\xi=\xi_{*}$.

To apply Theorem 10 to (104), we verify all the assumptions. Note that

$$
\begin{aligned}
& \delta=\epsilon^{1 / 2 n+2}, \quad r_{0}=\epsilon^{2 n+1 / 4 n+4}, \\
& s_{0}=s, \quad \sigma_{0}=\delta, \quad \epsilon_{0}=c \epsilon .
\end{aligned}
$$

Let

$$
E_{0}=\frac{\epsilon_{0} \Gamma\left(m_{0} / 2\right) \Gamma\left(\rho_{0}\right)}{\alpha r_{0}}=\frac{\epsilon^{2 n+3 / 4 n+4} \Gamma\left(m_{0} / 2\right) \Gamma\left(\rho_{0}\right)}{\alpha} .
$$

Thus when $\epsilon$ is small enough, $E_{0}$ is also small. Moreover, we have

$$
\begin{gathered}
\frac{\epsilon_{0}}{E_{0} \sigma_{0}}=\frac{\alpha \epsilon^{(2 n-1) /(4 n+4)}}{\Gamma\left(m_{0} / 2\right) \Gamma\left(\rho_{0}\right)} \leq \frac{1}{4}, \\
\frac{\epsilon_{0}}{r_{0} \sigma_{0}}=\epsilon^{(2 n+1) /(4 n+4)} \leq \frac{1}{16}, \\
\frac{E_{0}}{r_{0}}=\frac{\epsilon^{1 / 2 n+2} \Gamma\left(m_{0} / 2\right) \Gamma\left(\rho_{0}\right)}{\alpha} \leq 1 .
\end{gathered}
$$

So all the inequalities of (21) hold. Moreover, we have

$$
\left\||P \||_{m_{0}, \Delta_{r_{0}, s_{0} \times M_{0}}} \leq \epsilon_{0}=\frac{\alpha r_{0} E_{0}}{\Gamma\left(m_{0} / 2\right) \Gamma\left(\rho_{0}\right)} .\right.
$$

Since $|\widehat{N}( \pm \delta)| \leq 4 \epsilon_{0} \leq c \delta^{2 n+2}$, it follows that $\lambda( \pm \delta)=$ $\pm(A+\epsilon[a]) \delta^{2 n+1}+\widehat{N}( \pm \delta)$ must have a different sign for $\delta>0$ sufficiently small. Thus there exists a $\xi_{*} \in I$, such that $\lambda\left(\xi_{*}\right)=$ 0 . Moreover, we have $\left|\xi_{*}\right| \leq \delta=\epsilon^{1 /(2 n+2)}$ and $p_{*}=\left(\xi_{*}, 0\right) \in T$. Hence, by the transformation $x=\Phi\left(y, t ; p_{*}\right)$ the differential equation (103) at $\xi=\xi_{*}$ is changed to

$$
\dot{y}=A_{*} \Omega_{*} y+P_{*}\left(y, t, p_{*}\right)
$$

with $P_{*}\left(y, t, p_{*}\right)=O\left(y^{2}\right)(y \rightarrow 0)$. Therefore by the transformation $x=\Phi\left(y+\xi_{*}, t ; p_{*}\right)$ the system (5) is changed to the form of (17). Moreover, $x(t)=\Phi\left(\xi_{*}, t ; p_{*}\right)$ is an almostperiodic solution with the frequency $\omega$.

Since $\Phi\left(y+\xi_{*}, t ; p_{*}\right)=y+u_{*}(t ; p)+\xi_{*}$, we have $x=$ $y+u(t)$ with $u(t)=u_{*}(t ; p)+\xi_{*}$. Noting that $\left|\xi_{*}\right| \leq \epsilon^{1 /(2 n+2)}$, it follows that, for $p \in T,\|\| u \|_{0, s_{0} / 2}=O\left(\epsilon^{1 /(2 n+2)}\right)$. Thus, Theorem 9 is proved.

\section{Acknowledgments}

This work was partially supported by the National Natural Science Foundation of China (Grant nos. 10871117, 11171185) and SDNSF (Grant no. ZR2010AM013).

\section{References}

[1] N. N. Bogoljubov, A. Yu. Mitropolskii, and A. M. Samoilenko, Methods of Accelerated Convergence in Nonlinear Mechanics, Springer, New York, NY, USA, 1976, The Original Russian Edition: Metod Uskorennoj Schodimosti v Nelinejnoj Mechanike, Naukova Dumka, Kiev, Ukraine, 1969.

[2] J. Moser, "Convergent series expansions for quasi-periodic motions," Mathematische Annalen, vol. 169, no. 1, pp. 136-176, 1967.

[3] À. Jorba and C. Simó, "On quasi-periodic perturbations of elliptic equilibrium points," SIAM Journal on Mathematical Analysis, vol. 27, no. 6, pp. 1704-1737, 1996.

[4] À. Jorba and C. Simó, "On the reducibility of linear differential equations with quasiperiodic coefficients," Journal of Differential Equations, vol. 98, no. 1, pp. 111-124, 1992.

[5] J. Xu and S. Jiang, "Reducibility for a class of nonlinear quasiperiodic differential equations with degenerate equilibrium point under small perturbation," Ergodic Theory and Dynamical Systems, vol. 31, no. 2, pp. 599-611, 2011.

[6] J. X. Xu and J. G. You, "Reducibility of linear differential equations with almost periodic coefficients," Chinese Annals of Mathematics A, vol. 17, no. 5, pp. 607-616, 1996 (Chinese).

[7] H. Rüssmann, "On the one-dimensional Schrödinger equation with a quasiperiodic potential," Annals of the New York Academy of Sciences, vol. 357, pp. 90-107, 1980.

[8] J. Pöschel, "Small divisors with spatial structure in infinitedimensional Hamiltonian systems," Communications in Mathematical Physics, vol. 127, no. 2, pp. 351-393, 1990.

[9] A. M. Fink, Almost Periodic Differential Equations, vol. 377 of Lecture Notes in Mathematics, Springer, Berlin, Germany, 1974.

Thus Theorem 10 holds for (104). 


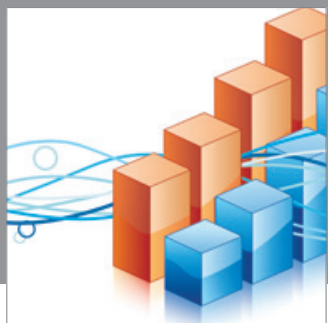

Advances in

Operations Research

mansans

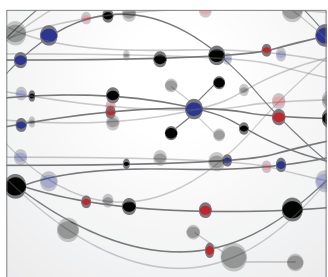

The Scientific World Journal
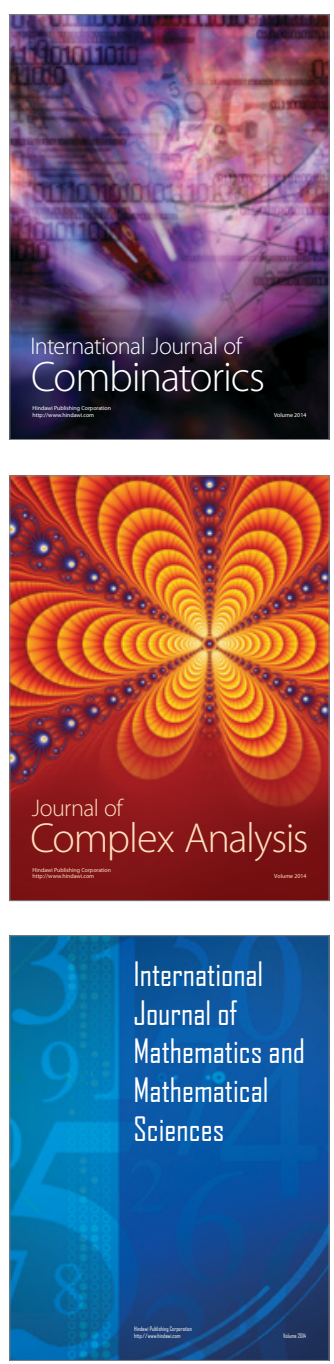
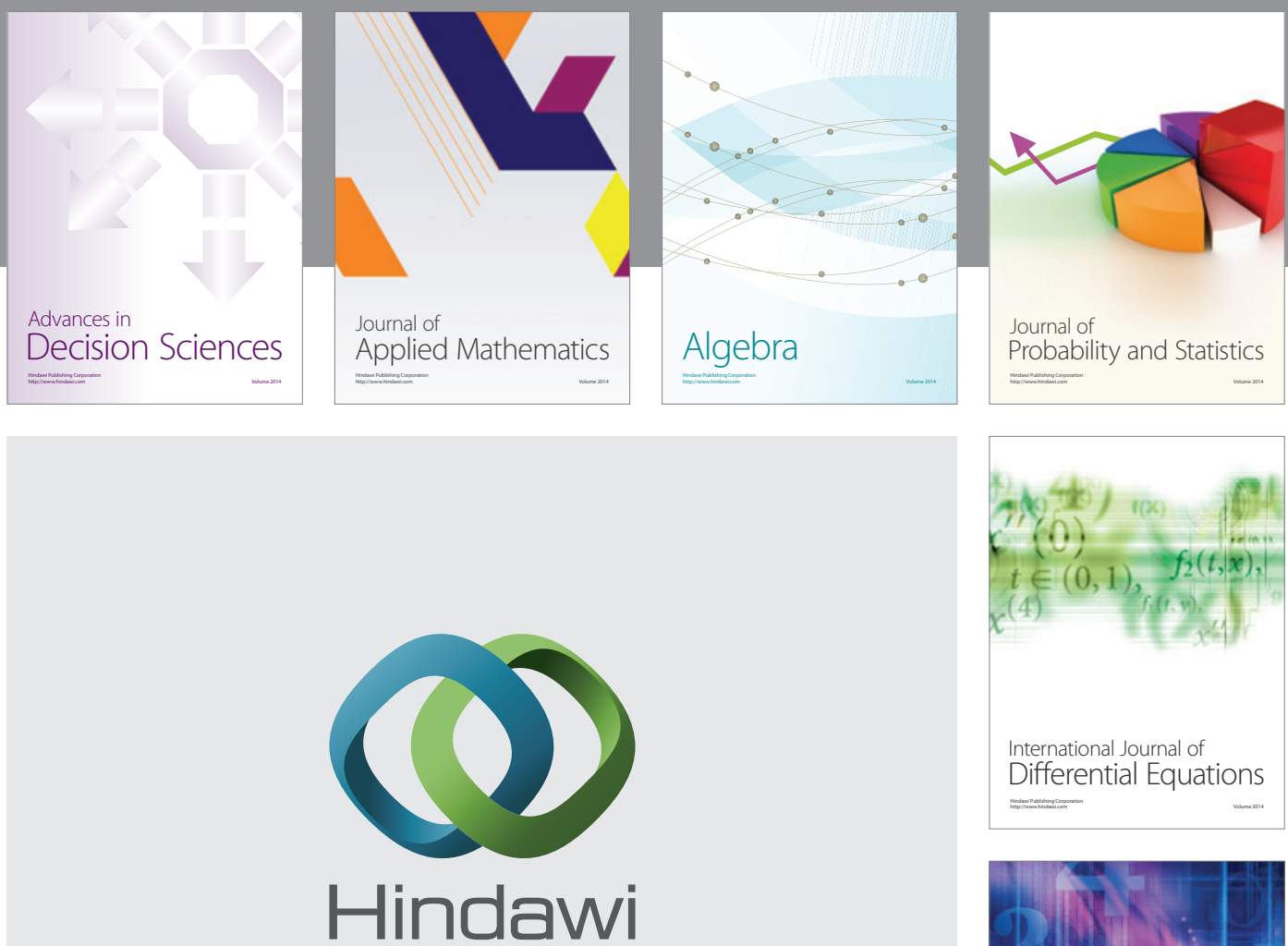

Submit your manuscripts at http://www.hindawi.com
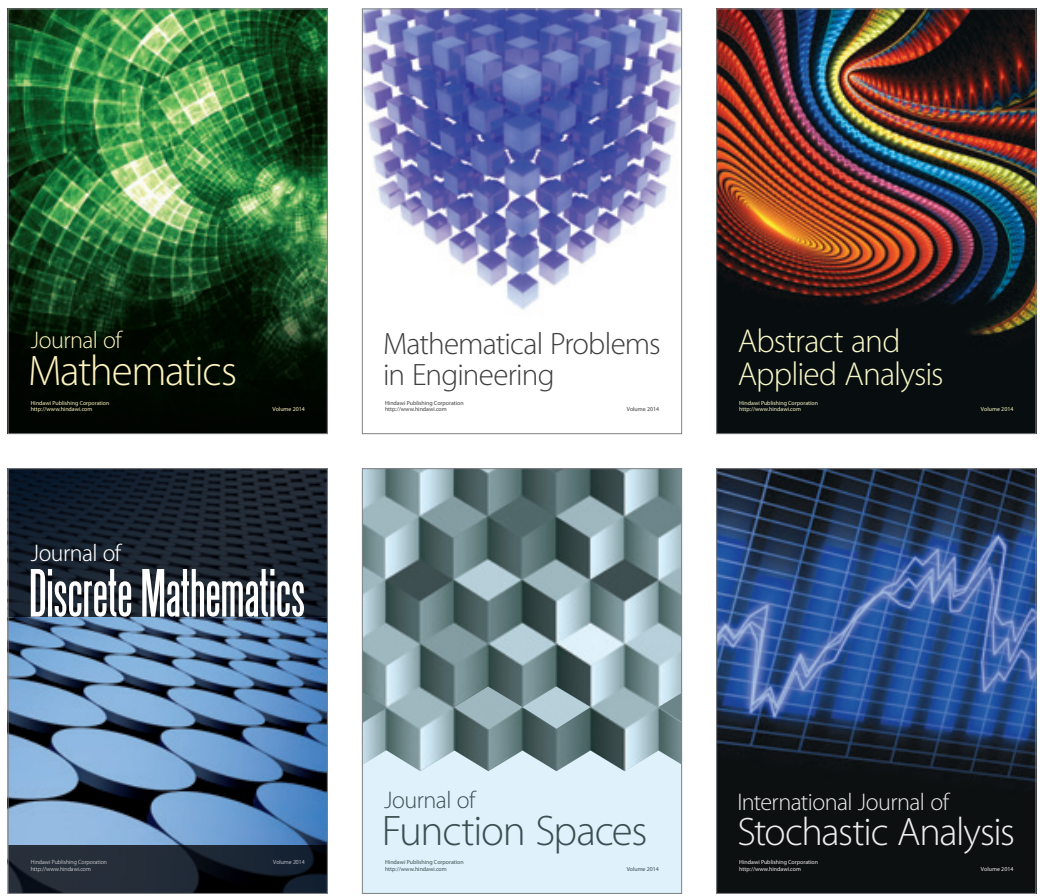

Journal of

Function Spaces

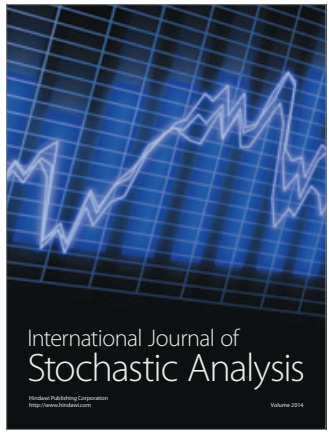

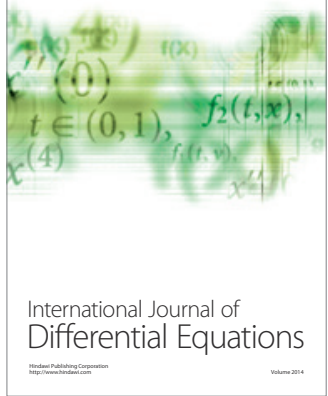
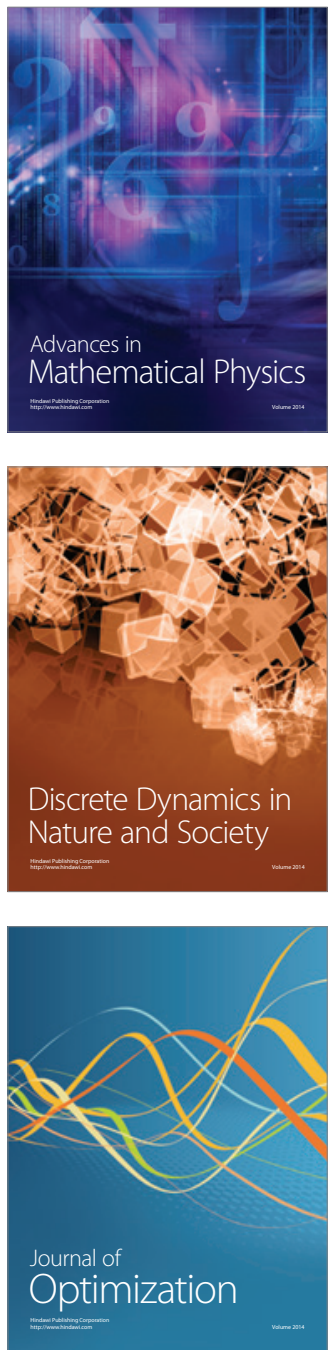